\title{
Variability in biological monitoring of organic solvent exposure. II Application of a population physiological model
}

\author{
P O DROZ, M M WU, W G CUMBERLAND \\ From the School of Public Health, University of California at Los Angeles, Los Angeles CA 90024, USA
}

\begin{abstract}
A physiological population model is used to study the variability associated with the biological monitoring of solvent exposure. The model consists of a combination of a physiological compartmental model and statistical simulation technique. Variable components considered are: exposure concentration, physical workload, body build, liver function, and renal function. The model is applied to six solvents: trichloroethylene, tetrachloroethylene, methylchloroform, benzene, toluene, and styrene. Biological indicators and air monitoring are compared as predictors of exposure, both external and internal (uptake, brain concentration, reactive metabolite formation). It appears that the choice of the best indicator depends on the type of exposure which is to be predicted. The effects of the various factors, environmental, physiological, or metabolic, are quantified and discussed. It is shown that fluctuation in exposure plays a large part in the final variability of biological indicator results. Further improvements and applications of this population model are considered.
\end{abstract}

The comparison of biological monitoring results with corresponding air monitoring data often discloses a substantial residual variability. ${ }^{1-3}$ The interpretation of the latter is the subject of much controversy and in many cases leads to the conclusion that biological monitoring can only be semiquantitative. Before drawing such conclusions, variability should be studied in greater detail in order to identify the factors responsible for it and its toxicological importance.

In a preceding paper we developed a population physiological model to describe groups of differing workers exposed in fluctuating industrial environments. ${ }^{4}$ It consists of a combination of a physiological compartmental model with statistical simulation techniques, allowing the random selection of some environmental and individual characteristics. This model is used here to describe and compare variability associated with biological monitoring for six organic solvents: trichloroethylene, tetrachloroethylene, methylchloroform, benzene, toluene, and styrene. It is also used quantitatively to identify the main factors responsible for this variability.
Biological monitoring can generally be used to reach two different objectives:

(A) estimate the external exposure (usually the air exposure if there is no significant skin absorption), both recent or long term, or

(B) estimate the internal exposure (exposure of the body or some part of it), both recent or long term.

On the other hand, biological monitoring may be applied in three types of general conditions:

(1) Only one worker is investigated, but repeatedly over time.

(2) A group of workers who are doing the same job or may be considered as having the same long term exposure is measured.

(3) A group of workers who are working at different processes or in different plants or both (different long term exposures) is considered.

The present paper considers for each solvent, the following questions:

(I) For a given condition $(1,2$, or 3$)$ and a given objective (A or B), which biological indicator is the best choice (solvent or metabolites in breath, blood, or urine collected at the end of shift or the next morning);

(II) In the same context $(1,2$, or 3 and $A$ or $B)$ how 
do the possible biological indicators compare with air monitoring (recent or long term);

(III) For a given biological indicator, should we worry about the characteristics of the individual workers (physical workload, body build, liver and renal functions) or of the workplaces (exposure variability) in interpreting the results.

\section{Methods}

\section{POPULATION MODEL DESCRIPTION}

The model used consists of seven physiological compartments. Solvent metabolism is simulated in the liver compartment by using the liver blood flow and the intrinsic metabolic clearance. The metabolite(s) formed are each distributed into a single volume of distribution and then excreted through the kidneys. Variability is simulated with the following five general concepts:

Exposure concentration with three components: between workers (long term), within worker between days, within worker within day.

Physical workload with two components: between workers (long term), within worker between days.

Body build based on two components; the body height and body weight or rather the Quetelet index (weight/ height $^{2}$ ).

Liver function with three components: the liver blood flow, the intrinsic metabolic clearance, and the metabolic ratio- that is, the variability between two parallel routes of metabolism.

Renal function with three components; the creatinine renal clearance and the urine formation times both for the end of shift and the next morning samples.

- Statistical distributions are used to describe each of the above random components. Their characteristics (shape, central tendency, and dispersion) were estimated from real data gathered on workers or healthy patients. ${ }^{4}$ For the liver function, uncertainties existed among the values to be used to describe the dispersion of the distributions. Therefore a range of values was considered. For the other components, the use of a single distribution appeared to be satisfactory.

A detailed description of the model, with mathematical relations, may be found in the preceding paper. ${ }^{4}$

\section{GENERAL STRATEGY FOR THE SIMULATIONS}

The exposure pattern simulated follows the normal work cycle: eight hours work interrupted by a one hour break, five days a week for several weeks. On the fifth week, Thursday and Friday mornings are chosen for monitoring. The following exposure indicators are calculated:

External exposure-Time weighted average expo-
Table 1 Definition of biological and exposure indicators

External exposure/air monitoring indicators

TWA-D $8 \mathrm{~h}$ TWA solvent concentration in air (Thursday)

TWA-W $\quad 40 \mathrm{~h}$ TWA solvent concentration in air (entire week)

Internal exposure indicators

ABS-D $\quad 8 \mathrm{~h}$ total solvent uptake (Thursday)

ABS-W $\quad 40 \mathrm{~h}$ total solvent uptake (entire week)

BRAIN-D $\quad 8 \mathrm{~h}$ TWA solvent brain concentration (Thursday)

BIO-W $40 \mathrm{~h}$ TWA formation rate of reactive metabolites (entire week)

Biological monitoring indicators

calv0 Breath solvent concentration $0.5 \mathrm{~h}$ postexposure

calv15 Breath solvent concentration $15 \mathrm{~h}$ postexposure

cven0 Venous blood solvent concentration $0.5 \mathrm{~h}$ postexposure

cven 15 Venous blood solvent concentration $15 \mathrm{~h}$ postexposure

bm10 Venous blood metabolite 1 concentration $0.5 \mathrm{~h}$ postexposure

bm20 Venous blood metabolite 2 concentration $0.5 \mathrm{~h}$ postexposure

bm115 Venous blood metabolite 1 concentration $15 \mathrm{~h}$ postexposure

bm215 Venous blood metabolite 2 concentration $15 \mathrm{~h}$

um10c Urinary excretion of metabolite 1 end of exposure (creat corr)

um20c Urinary excretion of metabolite 2 end of exposure

um115c Urinary excretion of metabolite $115 \mathrm{~h}$ postexposure (creat corr)

um215c Urinary excretion of metabolite $215 \mathrm{~h}$ postexposure $\frac{\bar{c}}{\omega}$ (creat corr)

usol0 Urinary solvent concentration end of exposure

usol15

Urinary solvent concentration $15 \mathrm{~h}$ postexposure

sure concentration on Thursday (recent, TWA-D) and of the entire week (long term, TWA-W);

Internal exposure -Uptake on Thursday (ABS-18 and during the entire week (ABS-W), time weighte average concentration of solvent in brain on Thursdaf (BRAIN-D), and time weighted average rate of form tion of reactive metabolites during the entire week (BIO-W).

Internal exposure indicators were chosen to describe recent and long term total uptakes as generg concepts widely used, recent solvent brain concentr tions as acute central nervous system indicators, and long term reactive metabolites formation to describe potential chronic metabolism mediated toxicity. The various exposure indicators are summarised in table $\overline{\text {. }}$.

Biological monitoring is carried out on twe occasions: just after the end of work on Thursday $(0.5 \mathrm{~h}$ postexposure for blood and breath, end of sh for urine) and on Friday before shift. For breath the solvent concentration is calculated whereas for blood and urine both the solvent and the metabolites afe calculated. All the biological indicators considered ate summarised in table 1.

The conditions simulated are summarised in table I corresponds to the simulation of one worker monf tored 50 times over time; II describes 200 workefs engaged in the same or similar jobs and therefore 
Table 2 Description of the simulations carried out

\begin{tabular}{|c|c|c|c|c|c|c|}
\hline \multirow[b]{2}{*}{ Parameters } & \multicolumn{6}{|c|}{ Conditions } \\
\hline & $I$ & $I I$ & $I I I$ & IIIa & $I I I b$ & IIIc \\
\hline $\begin{array}{l}\text { No of workers } \\
\text { Exposure between: }\end{array}$ & 1 & 200 & 200 & 200 & 200 & 200 \\
\hline $\begin{array}{l}\text { Workers } \\
\text { Between days } \\
\text { Within days } \\
\text { Physical workload between: }\end{array}$ & $\begin{array}{l}\mathbf{N} \\
\mathbf{M} \\
\mathbf{M}\end{array}$ & $\begin{array}{l}\mathbf{N} \\
\mathbf{M} \\
\mathbf{M}\end{array}$ & $\begin{array}{l}\mathbf{M} \\
\mathbf{M} \\
\mathbf{M}\end{array}$ & $\begin{array}{l}\mathbf{M} \\
\mathbf{M} \\
\mathbf{M}\end{array}$ & $\begin{array}{l}\mathbf{M} \\
\mathbf{H} \\
\mathbf{M}\end{array}$ & $\begin{array}{l}\mathbf{M} \\
\mathbf{M} \\
\mathbf{H}\end{array}$ \\
\hline $\begin{array}{l}\text { Workers } \\
\text { Between days } \\
\text { Body build } \\
\text { Liver function } \\
\text { Renal function }\end{array}$ & $\begin{array}{l}\mathbf{N} \\
\mathbf{Y} \\
\mathbf{N} \\
\mathbf{N} \\
\mathbf{N}\end{array}$ & $\begin{array}{l}\mathbf{N} \\
\mathbf{Y} \\
\mathbf{Y} \\
\mathbf{L} \\
\mathbf{Y}\end{array}$ & $\begin{array}{l}\mathbf{Y} \\
\mathbf{Y} \\
\mathbf{Y} \\
\mathbf{L} \\
\mathbf{Y}\end{array}$ & $\begin{array}{l}\mathbf{Y} \\
\mathbf{Y} \\
\mathbf{Y} \\
\mathbf{H} \\
\mathbf{Y}\end{array}$ & $\begin{array}{l}\mathbf{Y} \\
\mathbf{Y} \\
\mathbf{Y} \\
\mathbf{L} \\
\mathbf{Y}\end{array}$ & $\begin{array}{l}\mathbf{Y} \\
\mathbf{Y} \\
\mathbf{Y} \\
\mathbf{L} \\
\mathbf{Y}\end{array}$ \\
\hline
\end{tabular}

$\mathbf{N}=$ No variability; $\mathbf{Y}=$ variability at the level defined in the development of the model; $\mathbf{M}=$ medium variability for exposure

GSD = 2.0; $\mathbf{H}=$ high variability, for exposure GSD $=3.0$, for liver function high blood flow and metabolic ratio variability;

$\mathbf{L}=$ low variability in liver function (low hepatic blood flow and metabolic ratio variability).

having identical long term exposure; and III concerns 200 workers doing various jobs, possibly in different plants. Conditions I to III have low metabolic variability (for metabolic ratio and liver blood flow) and a medium exposure variability (GSD $=2.0$ for the three components). Three more protocols were considered to investigate the effect of higher metabolic and exposure variabilities. Condition IIIa is identical to III but with high metabolic variability; IIIb is the same as III but with higher within worker between days variability (GSD $=3.0$ ); and IIIc describes higher within worker within day exposure variability (GSD $=3.0$ ).

The model is applied to six widely used volatile chemicals and their metabolites (see table 3 ).

\section{STA TISTICAL METHODS}

The results of the model are organised according to a case structure. For each case (worker or measurement for a single worker), the model stores all exposure and biological indicators and characteristics of the worker and the environment. Three main aspects were considered for analysis: (1) comparison of biological/air indicators as predictors of exposure (external and

Table 3 Solvents and their metabolites

\begin{tabular}{|c|c|c|}
\hline \multirow[b]{2}{*}{ Solvent } & \multicolumn{2}{|l|}{ Metabolites } \\
\hline & Abbreviation & Model code \\
\hline Trichloroethylene & Trichloroacetic acid TCA & Metabolite 1 \\
\hline $\begin{array}{l}\text { Tetrachloroethylene } \\
\text { Methylchloroform }\end{array}$ & $\begin{array}{l}\text { Trichloroacetic acid TCA } \\
\text { Trichloroacetic acid TCA } \\
\text { Trichloroethanol TCE }\end{array}$ & $\begin{array}{l}\text { Metabolite } 2 \\
\text { Metabolite } 1 \\
\text { Metabolite } 2\end{array}$ \\
\hline $\begin{array}{l}\text { Benzene } \\
\text { Toluene }\end{array}$ & $\begin{array}{l}\text { Phenol } \\
\text { Hippuric acid HA } \\
\text { O-cresol }\end{array}$ & $\begin{array}{l}\text { Metabolite } 2 \\
\text { Metabolite } 2 \\
\text { Metabolite } 1\end{array}$ \\
\hline Styrene & $\begin{array}{l}\text { Mandelic acid MA } \\
\text { Phenylglyoxylic acid PGA }\end{array}$ & $\begin{array}{l}\text { Metabolite } 2 \\
\text { Metabolite } 1\end{array}$ \\
\hline
\end{tabular}

internal), (2) influence of environmental variability on the quality of the biological indicators as predictors of exposure, and (3) effect of individual factors on the biological indicators.

(1) Biological and air indicators $(X)$ are compared as predictors of the various exposure indices (Y). Scatterplots of $\mathrm{Y}$ as a function of $\mathrm{X}$ indicated a variance increasing with $\mathrm{X}$. To stabilise the residuals, log-log plots were considered and finally the following model was used:

$$
Y_{i}=A^{*} \mathbf{X}_{i}^{B} * e^{e^{i}}
$$

where $\varepsilon_{\mathrm{i}}$ is an error term normally distributed, and $\mathbf{A}$ and $B$ are constants. The residual mean square $s^{2}\left(\varepsilon_{i}\right)$ is estimated by linear regression of $\ln (Y)$ over $\ln (X)$. To obtain a meaningful indicator of the residual variability, the $\mathrm{s}^{2}\left(\varepsilon_{\mathrm{i}}\right)$ is transformed in the linear scale by calculating the following coefficient of variation:

$$
\mathrm{CV}=\left[\exp \left(\mathrm{s}^{2}\left(\varepsilon_{\mathrm{i}}\right)-1\right]^{1 / 2}\right.
$$

This CV expresses the magnitude of the uncertainty in the prediction of $\mathrm{Y}$ with $\mathrm{X}$. They are calculated for each possible biological indicator with each exposure concept in all conditions presented in table 2 .

(2) The evaluation of the contribution of environmental variability to biological variability is estimated by comparing CVs obtained in conditions II (constant exposure), III (between workers), IIIb (between days), and IIIc (within days).

(3) The effects of individual factors are estimated using the following model with $\mathrm{X}=$ exposure and $\mathrm{Y}$ $=$ biological indicators:

$$
Y_{i}=A^{*} X_{i}^{B *} e^{b j i j} e^{e i}
$$

where $\mathrm{f}_{\mathrm{j}}$ are individual factors: body build expressed as Quetelet index, physical workload as pulmonary ventilation, liver function using the three components blood flow, intrinsic metabolic clearance, and metabolic ratio, and renal function based on the creatinine clearance. The $b_{j}$ are the corresponding slopes and they are estimated by multiple linear regression analysis of $\ln (Y)$ on $\ln (X)$ and the untransformed $f_{j}$. To obtain a useful estimator of the effects of individual factors, the slopes bj are transformed back to the linear scale using the formula:

$$
F=\mathrm{e}^{\mathrm{bj} * 2 \mathrm{sj}}
$$

where $\mathrm{s}_{\mathrm{j}}$ is the standard deviation of the factor $\mathrm{fj}$, and $\mathrm{F}$ is a multiplicative factor indicating the effect on the biological indicator, when the individual factor is changed from its mean to two standard deviations higher. 


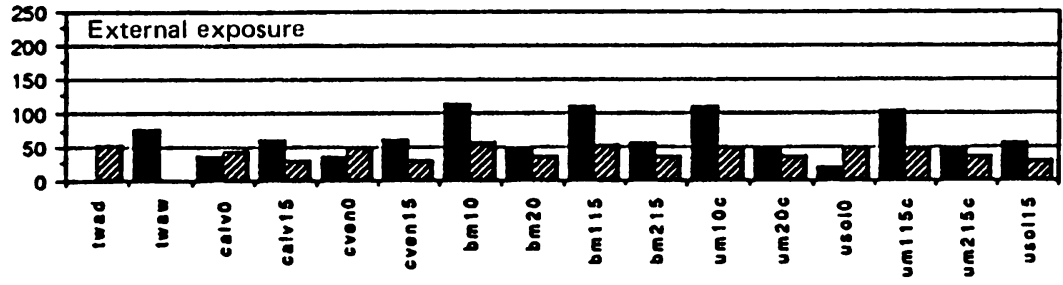

TWAD

TWAW
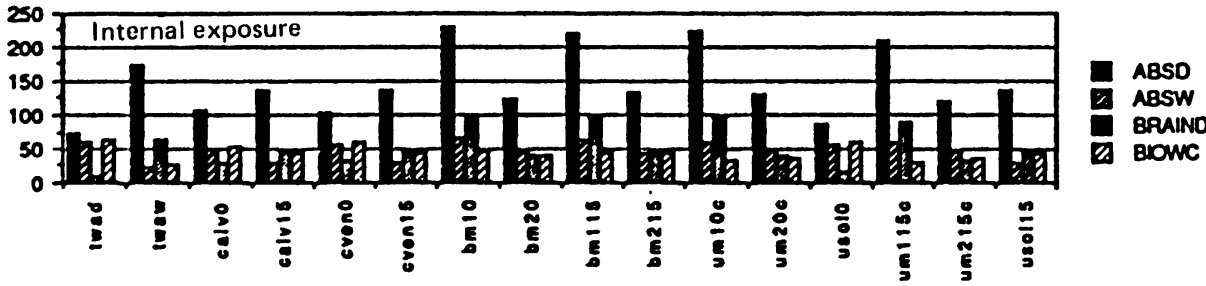

Fig 1 Trichloroethylene: comparison of biological and air monitoring indicators as predictors of external and internal exposure in condition III. Ordinate is coefficient of variation in per cent.

\section{Results and discussion}

COMPARISON OF BIOLOGICAL/AIR INDICATORS

Various biological indicators for a given solvent are compared on the basis of reliability to predict the exposure concepts presented in table 1. Biological indicators are similarly compared with the air monitoring indicators (TWA-D, TWA-A). The basis for comparison is the CV calculated on the residual variability. Figures 1 to 6 present the results obtained for the six solvents in the case of condition III-that is, 200 workers exposed in various jobs and industries. They show the CV obtained for the prediction of both the external exposure (TWA-D, TWA-W) and the internal exposure (ABS-D, ABS-W, BRAIN-D, BIO-
W). The symbols are described in table 1. For pheno요, o-cresol, and hippuric acid, the $\mathrm{CV}$ are calculated for $\overrightarrow{\vec{a}}$ mean exposure of the group of 200 workers at the TLV: $10 \mathrm{ppm}$ for benzene and $100 \mathrm{ppm}$ for toluene Different mean exposures would slightly modify the results, a lower exposure producing a higher $\mathrm{CV}$ owing to the natural background of the indicator.

External exposure prediction-Recommended tio logical limit values are often extrapolated frên or made equivalent to, airborne concentrations. Therefore, the comparative reliability of differem indicators to predict external exposure is importanto The air monitoring indicators (TWA-D, TWA-W) are the best predictors of external exposure, although TWA-D gives a CV of $54 \%$ for the prediction of long
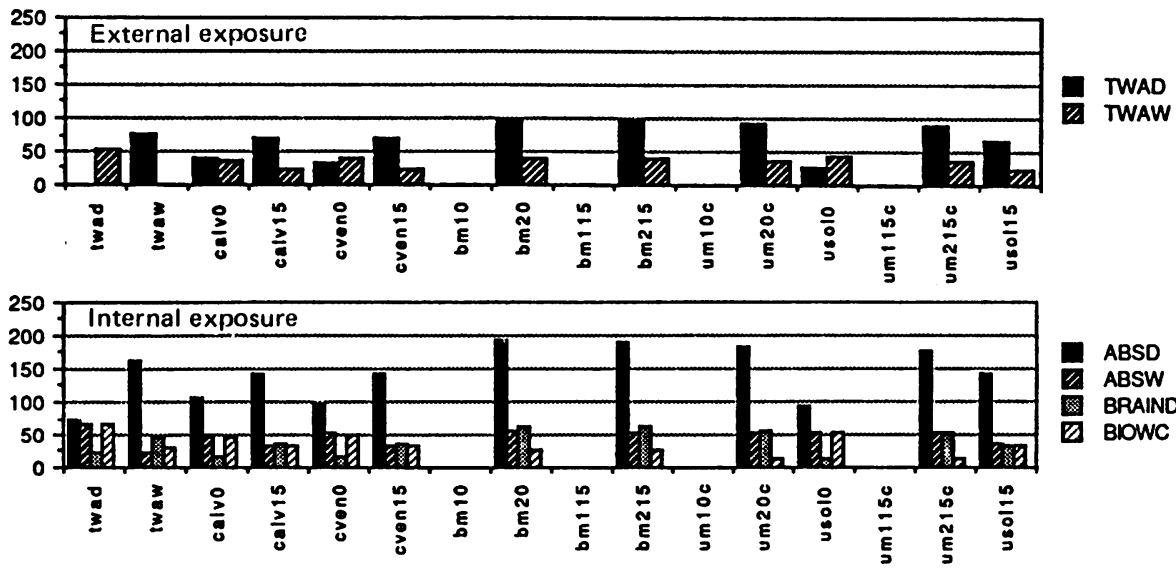

Fig 2 Tetrachloroethylene: comparison of biological and air monitoring indicators as predictors of external and internal exposure in condition III. Ordinate is coefficient of variation in per cent. 


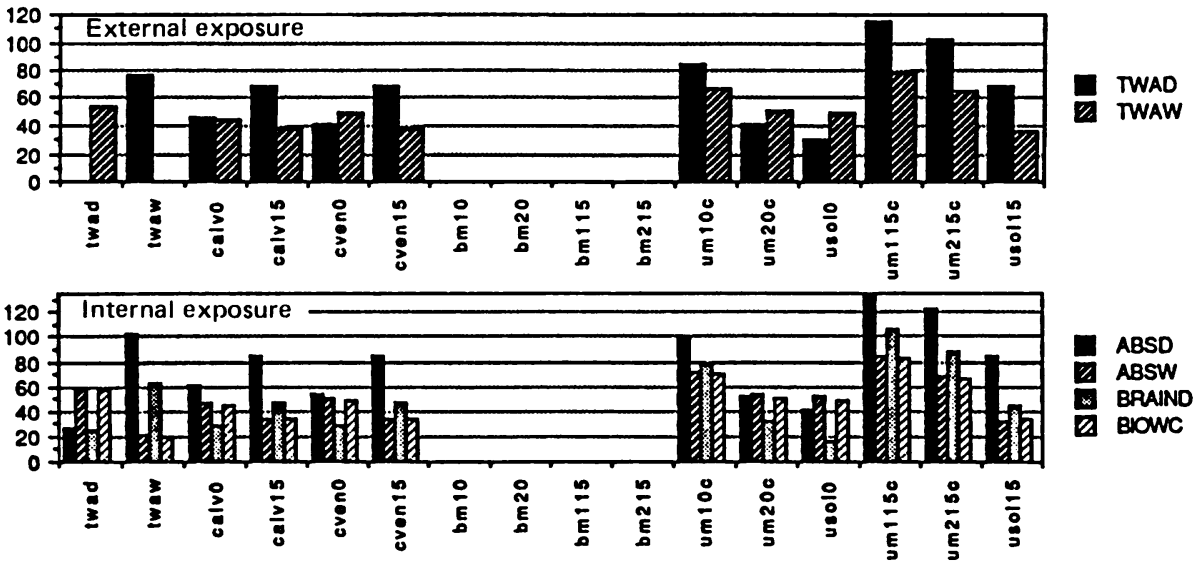

Fig 3 Methylchloroform: comparison of biological and air monitoring indicators as predictors of external and internal exposure in condition III. Ordinate is coefficient of variation in per cent.

term exposure in the conditions of medium environmental fluctuations (GSD $=2 \cdot 0$ ). This is a figure that long term biological indicators may be compared with. Examination of the external exposure portions of figs 1 to 6 allows biological indicators of recent and long term exposure to be identified. The CVs qualitatively follow the apparent half life of the indicators at the time of sampling: solvent in breath, blood, or urine at the end of shift and short half life metabolites in blood and urine indicate preferentially recent exposure; on the other hand, solvent in breath, blood, or urine before shift and long half life metabolites show more aspects of long term exposure. This was to be expected as long as half life chemicals tend to integrate repeated exposures. ${ }^{7}$ The differences in the CVs would still be more pronounced for conditions with higher environmental variabilities (IIIb and IIIc) as shown in figs 7 and 8 for, respectively, tetrachloroethylene and styrene. Increasing environmental variabilities between workers (II to III), between days (III to IIIb), within days (III to IIIc), always increases variability in biological indicators. This effect is the most pronounced for long half life indicators used as predictors of recent exposure when the between days variability is increased. Similar results are obtained for the four other solvents studied. The comparison, in figs 1 to 6 , of biological indicators' CVs of extensively (trichloroethylene, benzene, toluene, styrene) and slowly metabolised solvents (tetrachloroethylene, methylchloroform) shows that there is the following
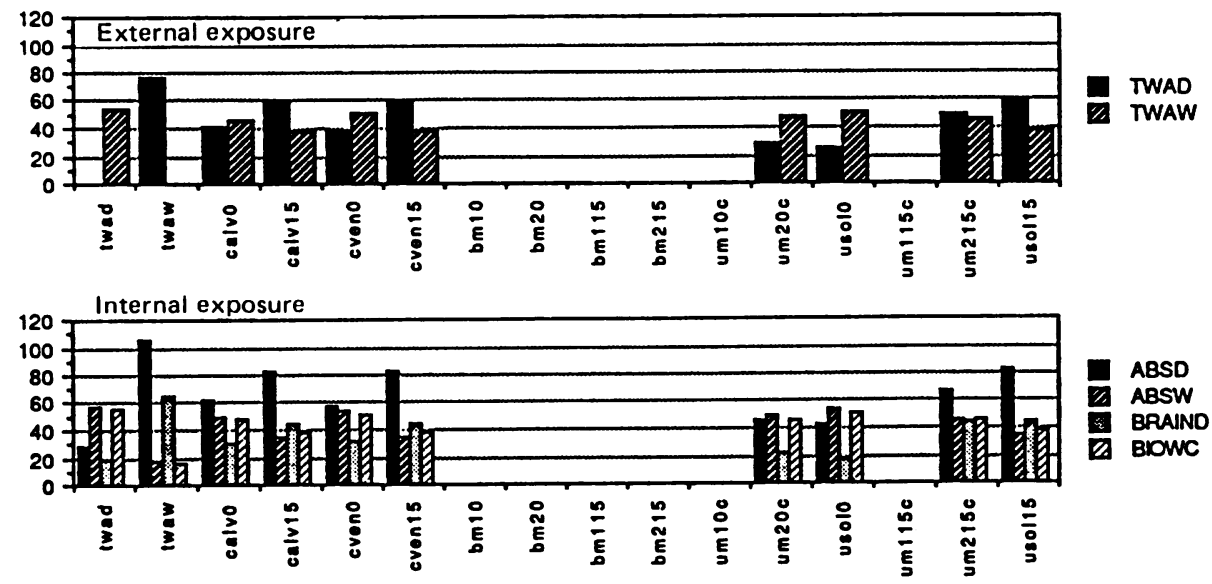

Fig 4 Benzene: comparison of biological and air monitoring indicators as predictors of external and internal exposure in condition III. Ordinate is coefficient of variation in per cent. 


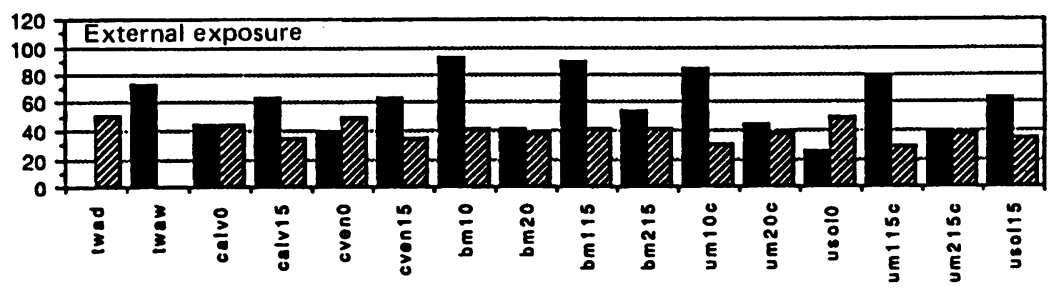

TWAD

TWAW
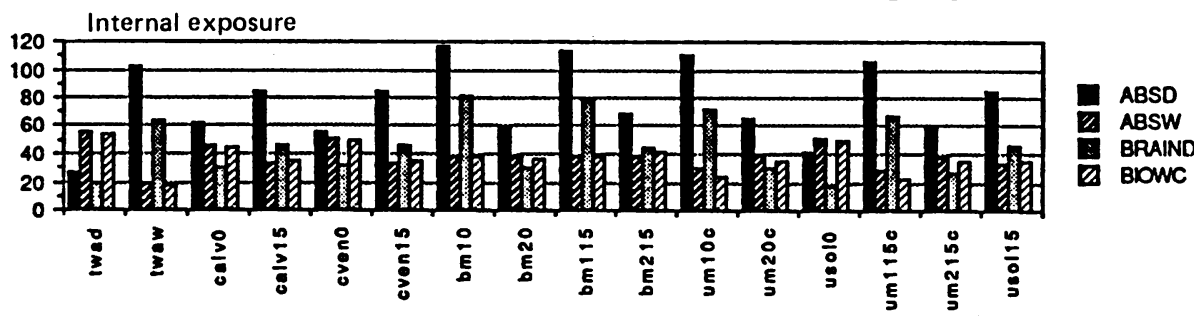

Fig 5 Toluene: comparison of biological and air monitoring indicators as predictors of external and internal exposure in condition III. Ordinate is coefficient of variation in per cent.

tendency: indicators based on intact solvent of slowly metabolised chemicals or on metabolites of extensively biotransformed solvents are generally the least variable. When compared with one day air monitoring performance (TWA-D), biological monitoring appears to be as good or better a predictor of long term exposure.

Internal exposure prediction: Four concepts of internal exposure are presented in figs 1 to 6 . The total uptake (ABS-D, ABS-W) is presented here because it is often used by others to describe internal exposure. ${ }^{8}$ It has, nevertheless, little toxicological importance. Of greater importance is the concentration of solvent in a target organ, the brain, for example (BRAIN-D), or an index of active metabolites formation (BIO-W).
The first concept is applied to the recent exposure as intact solvent toxicity is often believed to be of a short term nature; the second internal exposure descriptor used to represent long term chronic toxicity. From figs 1 to 6 , it is obvious that most of the biological indicators are relatively poor predictors of recerfe uptake (ABS-D) when compared with air monito $B$ n $\subseteq$ (TWA-D) or to the prediction of other exposire concepts. Biological indicators tend to predict 䗆

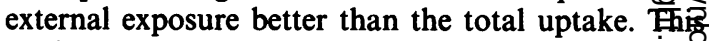
tendency is even more clear in the case of slowl\$ biotransformed solvents, such as tetrachloroethylen and methylchloroform for which the CVs may reach more than $200 \%$. The situation for the prediction of long term uptake is somewhat more favourable to
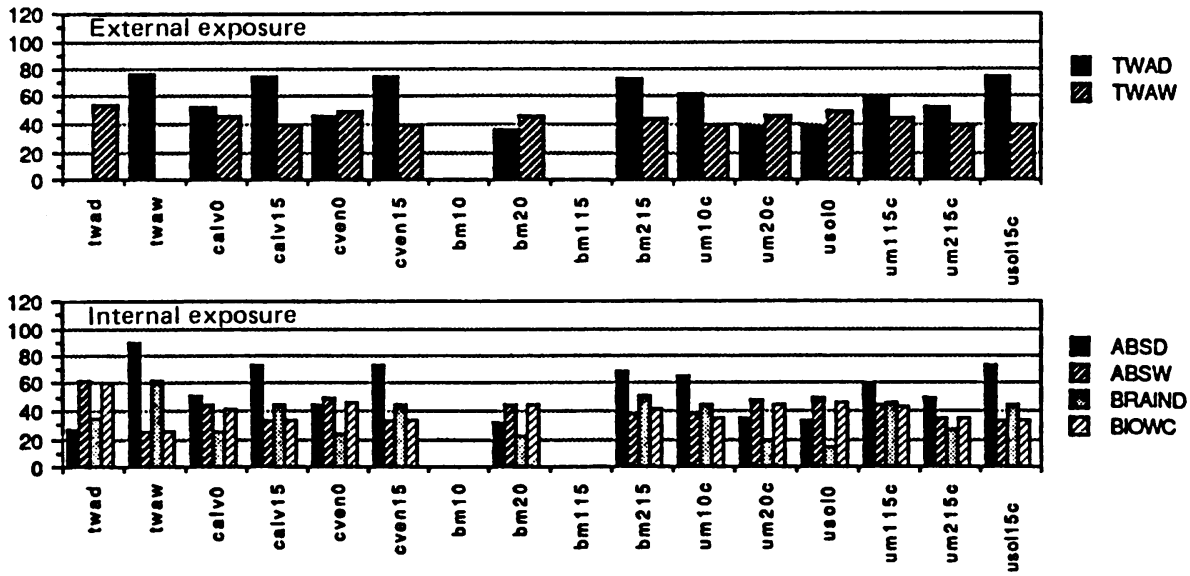

Fig 6 Styrene: comparison of biological and air monitoring indicators as predictors of external and internal exposure in condition III. Ordinate is coefficient of variation in per cent. 

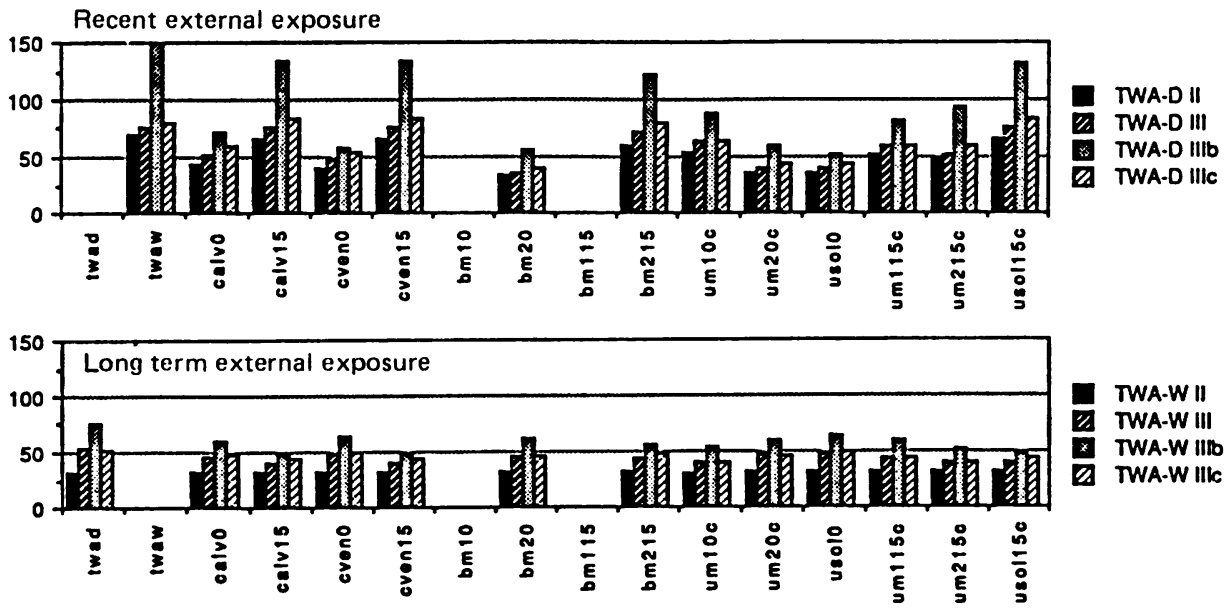

Fig 7 Tetrachloroethylene: comparison of biological and air monitoring indicators as predictors of external exposure for conditions II, III, IIIb, and IIIc. Ordinate is coefficient of variation in per cent.

biological indicators having long half lives, owing to their capability to integrate repeated fluctuating exposures. When looking at the prediction of brain recent concentrations (BRAIN-D), biological indicators are more efficient as shown by the lower CVs presented in figs 1 to 6 . Nevertheless, in most cases single day air monitoring gives as good, if not better results. For the prediction of the formation of long term active metabolites (BIO-W), the situation is more favourable to biological indicators with long half lives which are usually much better predictors than single day air monitoring (TWA-D) and even long term air monitoring (TWA-W). This is particularly true in the case of slowly biotransformed solvents for metabolites in blood and urine.

Trichloroethylene-TCA is the best indicator of long term exposure, followed by the solvent in breath, blood, or urine before shift. Blood metabolites are slightly less interesting than in urine, but in practice the situation might differ as the model does not take into account the effect of urine flow on renal clearance.' For the prediction of recent exposure, the solvent in urine collected at the end of shift seems to be best. This is in agreement with the excellent correlations obtained for other solvents. ${ }^{10}$ This biological indicator is also an excellent predictor of recent brain
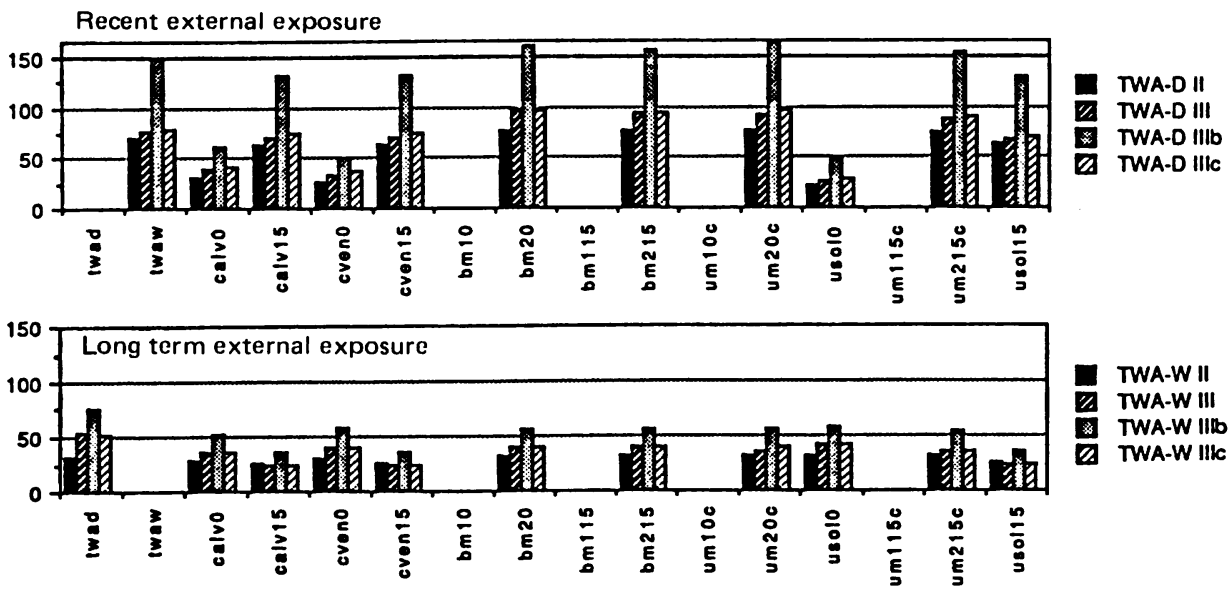

TWA-W II

Fig 8 Styrene: comparison of biological and air monitoring indicators as predictors of external exposure for conditions II, III, IIIb, and IIIc. Ordinate is coefficient of variation in per cent. 
concentration (BRAIN-D); all other biological indicators are less efficient than air monitoring (TWA-D). For the estimation of the rate of formation of active metabolites, TCA in urine seems to be the best indicator.

Tetrachloroethylene-The recent exposure is best estimated using measurements of the solvent in urine, blood, or breath at end of shift. The same indicators sampled before shift and TCA in blood or urine are all good predictors of long term exposure and all are better than single day air monitoring. The recent brain concentration is well estimated by end of shift breath, blood, or urine solvent concentrations. TCA in urine, possibly also in blood, is an excellent predictor of reactive metabolite formation, much better than both single day or entire week air monitoring.

Methylchloroform-The solvent concentration in urine at the end of shift is here again the best biological predictor of recent exposure, followed by the concentrations in breath or blood at the same time. The same indicators sampled before shift are the best long term exposure predictors, better than metabolites and single day air monitoring. The latter is nevertheless the best indicator of recent brain concentration, the best biological indicator being the urinary solvent concentration at the end of shift. The formation rate of reactive metabolites seems to be well predicted by urinary TCA excretion.

Benzene-Either the solvent or phenol in urine seems to be the best biological indicators of recent exposure. For long term exposure, the indicators based on the solvent itself appear to be slightly better than phenol. For the rate of formation of reactive metabolites, all the biological indicators show similar performances, with only a small improvement when .compared with single day air monitoring.

Toluene-For recent exposure monitoring, the solvent excreted in urine again seems to be the best, followed in order by hippuric acid and venous and breath solvent concentrations at the end of shift. This agrees with that found in field studies. ${ }^{10}$ The prediction of long term exposure is better if done again by measuring the solvent concentrations before shift. The brain concentration is well predicted by single day air monitoring or by the solvent in urine. Toluene in breath, blood, or urine are good global predictors of reactive metabolite formation. In this respect o-cresol shows quite poor results globally, but it might very well be a good indicator of a specific reactive metabolite (epoxide).

Styrene-Mandelic acid (MA) and styrene in urine are good indicators of recent exposure. For long term exposure all biological indicators seem to behave similarly, with not much improvement when compared with single day air monitoring. Brain concentration is well predicted by MA in blood or urine or by the solvent in breath, blood, or urine (end of shiftt. Biological indicators based on the solvent in brea唯, blood, or urine, or on MA in urine are satisfactory predictors of reactive metabolite formation whg sampled before shift.

The results presented in figs 1 to 6 have all been obtained with a low metabolic variability (condition III). CVs calculated based on a high metabowic variability (condition IIIa) give similar results för slowly metabolised solvents. In extensively biotrangformed chemicals only a slight increase in CVs was observed, mainly for the biological indicators based on the intact solvent. The small effect of the variability of liver function on the CVs to the biological indicators is probably because the CVs are only relat in part to individual variability.

ENVIRONMENTAL VERSUS INDIVIDUAL SOURCES户̈ OF VARIABILITY

The variability in the results of biological monitoriog may be produced both by fluctuations in exposure and by individual physiological or metabolic variabilif. Figure 9 shows for two solvents a comparison of the CVs of biological indicators for the prediction of external exposure in two cases: for one worker (con tion I) repeatedly monitored over time and for 290 workers in the same environment (condition II) monitored once each only. For tetrachloroethylene, thege are negligible differences only between the two an indication of a low contribution of physiologicaliamd metabolic characteristics to the global variabilit the case of styrene the differences are somewhat larg especially for long term exposure prediction. The foor other solvents show comparable results, methychloroform being similar to tetrachloroethylene, be $\vec{f}-$ zene, toluene, and trichloroethylene looking more like the extensively metabolised styrene. The results presented in figs 7 and 8 also confirm the importance af fluctuations in exposure: an increase in environmental variability produces an increase in the CVs of the biological indicators.

\section{PHYSIOLOGICAL AND METABOLIC SOURCES OF} VARIABILITY

Once the choice of a specific biological indicator $\dot{\text { os }}$ made, based on its CV and other criteria, it is important to consider which individual factors coudd affect the results obtained. The factors considered here are: physical workload, body build, liver function (perfusion and enzymatic function), and renal funotion. The results obtained are summarised in figs and 11. The effect of each factor on each biological indicator is quantified by the multiplicative factor $(F)$ produced by a change of 2 SDs of the individual parameter. If approximately normally distributed ag of 2 means that in most cases $(95 \%)$ one may expegf 

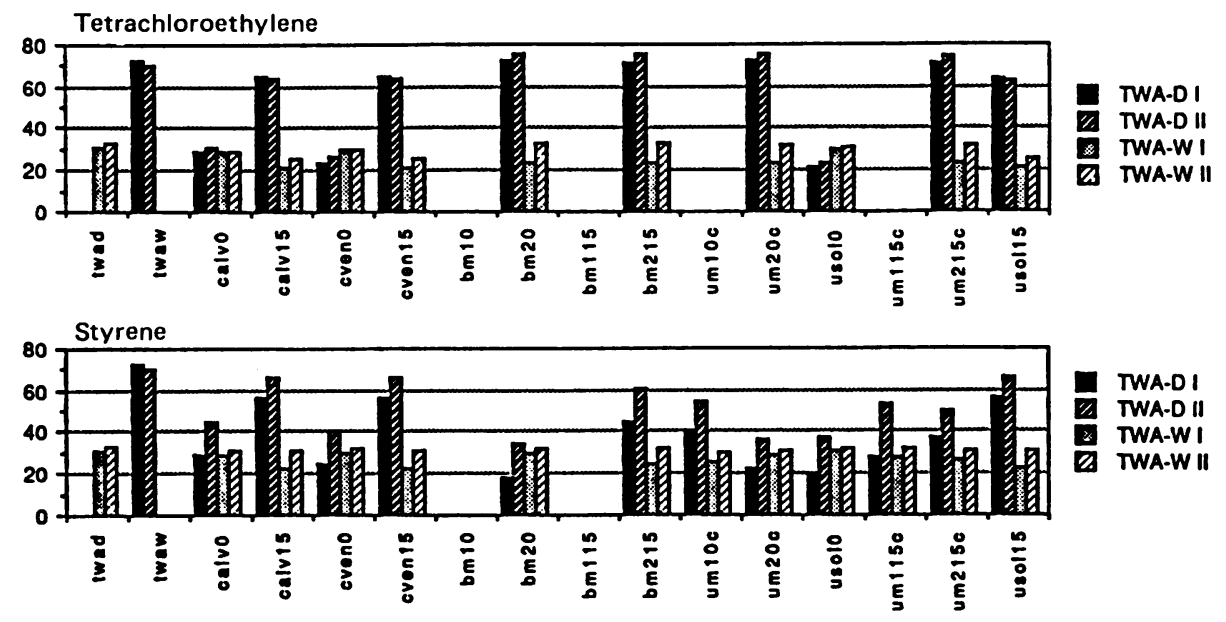

Fig 9 Environmental versus individual variability. Comparison of biological and air monitoring indicators as predictors of external exposure for conditions I (1 worker) and II (200 workers). Ordinate is coefficient of variation in per cent.

the biological indicator to vary within 0.5 and 2 times its mean value. A F of $1 \cdot 1(10 \%$ effect $)$ or below is not significant and may be considered as having no effect. The results presented in figs 10 and 11 were calculated for condition III but identical data are obtained by multiple linear regressions in other conditions (II, IIIb, IIIc). From figs 10 and 11, one may see that the effects of the individual characteristics depend both on the solvent and the biological indicator considered.

Trichloroethylene-Physical workload has a noticeable effect on all the biological indicators, being slightly more pronounced for the unchanged solvent than for its metabolites. Body build, as expected, influences mainly the solvent concentrations in breath, blood, or urine sample before shift but some effects may also be seen on the indicators based on the metabolites. The liver function has only a moderate effect, except for breath at the end of shift. Renal function is most important for TCA (metabolite 1) and TCE before shift in blood but not for urinary excretion. This is to put it in relation to the slow excretion rates of TCA and also of TCE before shift.

Tetrachloroethylene-Individual factors have a small impact on biological indicators for this solvent when compared with trichloroethylene. Physical workload has a general but low effect and body build plays no important part. The enzymatic function has a large impact on TCA in blood and urine but not on the unchanged solvent; this is compatible with the fact that tetrachloroethylene is very slowly metabolised. As for trichloroethylene, renal function has an important influence on blood TCA but not on its excretion in urine.
Methylchloroform behaves similarly to tetrachloroethylene. The main factors are the enzymatic and renal functions, which have much influence on TCA and somewhat less on TCE. Again, metabolites in blood are more affected than metabolites in urine by the renal function. Physical workload has a slightly higher effect than for tetrachloroethylene on the solvent in breath, blood, or urine sampled before shift.

Benzene-For this chemical, physical workload and body build are the dominant factors, affecting mainly the solvent in breath, blood, or urine sampled before shift. The liver and renal clearances have generally small effects on the biological indicators. For phenol (metabolite 2) urinary excretion, the natural background of this metabolite is the main source of variability which does not come out in the present analysis.

Toluene-Physical workload is the main contributor to the variability. As with benzene, body build is important for the unchanged solvent sampled before shift. Liver perfusion also has some importance for the unchanged solvent but not for metabolites. O-cresol is affected by the enzymatic function which includes the metabolic ratio: small changes in the metabolic ratio produce important differences in o-cresol (minor metabolite) but negligible ones in hippuric acid (major metabolite). Furthermore, as with benzene, the metabolites are also largely conditioned by the variability of the natural background.

Styrene-Among the six solvents studied, styrene is the most affected by physical workload with factors ranging systematically from 1.5 to 2.0 . The effect of body build is mostly restricted to the biological 

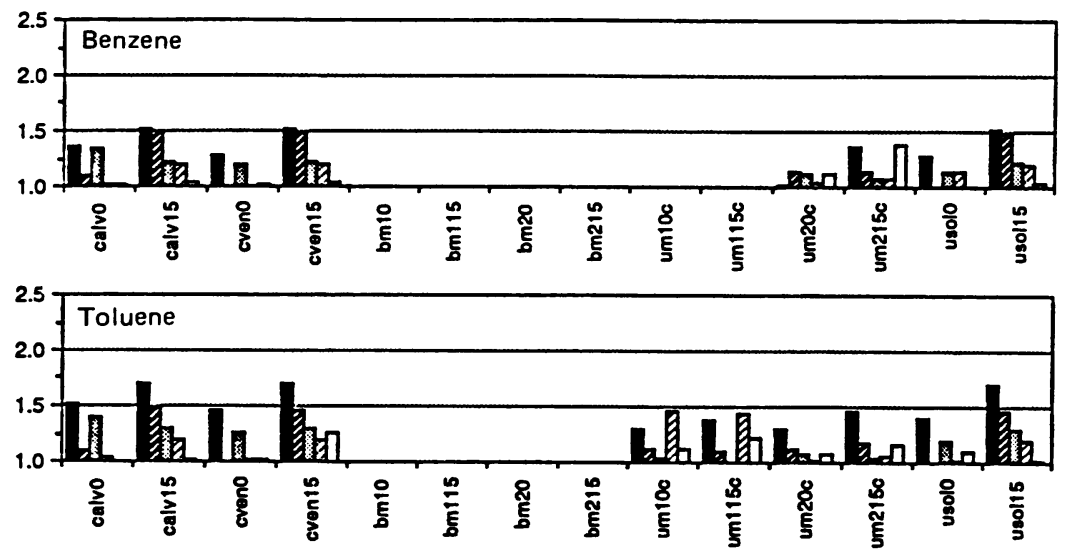

Workload Body build Liver flow Liver enzymes $\square$ Renal function

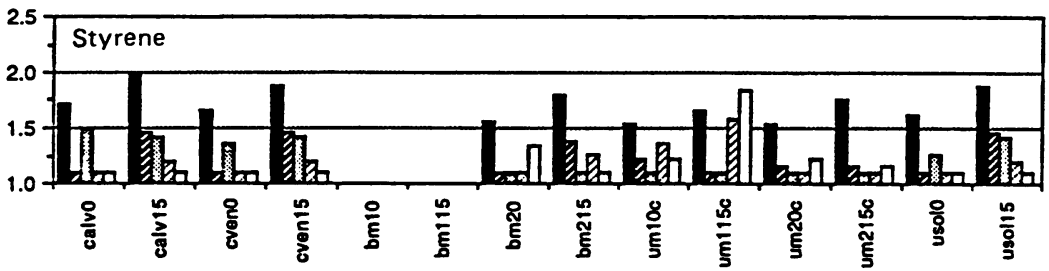

Fig 10 Influence of individual factors on levels of biological indicators. Ordinate is multiplicative factor $F$.
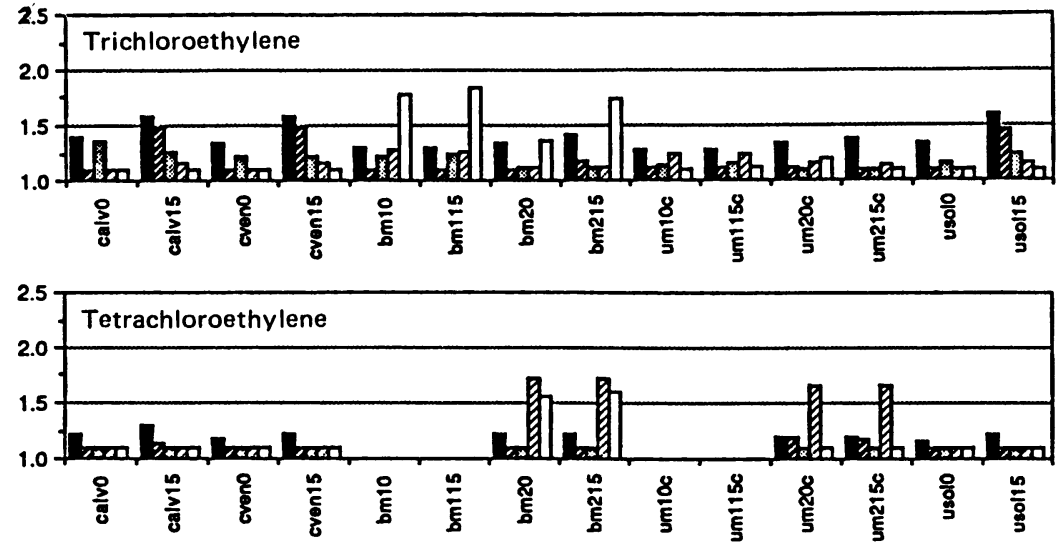

Workload

2 Body build

Liver blood

Liver enzymes

Renal function

Fig 11 Influence of individual factors on levels of biological indicators. Ordinate is multiplicative factor $F$. 

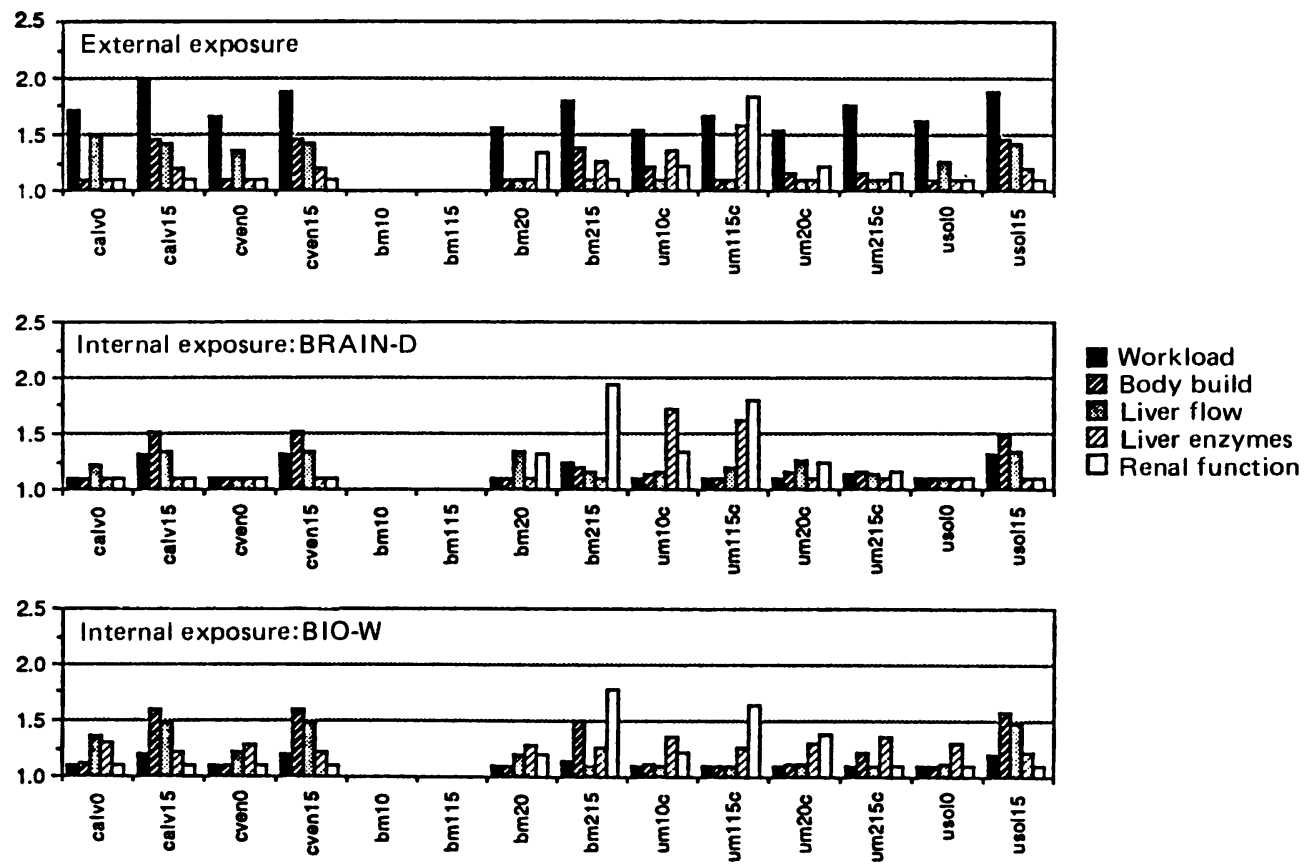

Fig 12 Styrene: influence of individual factors on level of biological indicators. Comparison of results obtained while predicting external exposure (TWA-D or TWA-W) and internal exposure $(B R A I N-D, B I O-W)$. Ordinate is multiplicative factor $F$.

indicators based on the unchanged solvent sampled before shift. MA in blood before shift is an exception, probably because its level then is mainly conditioned by the rate of formation from styrene. Among the liver function parameters, the liver perfusion exclusively influences unchanged solvent indicators, but the enzymatic function plays a part mostly in the metabolites, and more particularly in the phenylglyoxylic acid (PGA) levels, as changes in the enzymatic function will modify the ratio between excreted and biotransformed MA.

Figures 10 and 11 may be used to help understand differences between results obtained from air and biological monitoring programmes. They show the impact of individual factors on the correlation of biological indicators with external exposure. When using biological monitoring to estimate internal exposure, the relative importance of those factors completely changes, as shown in fig $\mathbf{1 2}$ for styrene. It summarises the same factors as in figs 10 or 11 while correlating with external exposure and internal exposure: recent mean brain concentration and long term average rate of reactive metabolite formation. For example, the effect of physical workload disappears almost completely, whereas the importance of renal function increases. The sign and magnitude of these modifications depend on the toxicological implications of the factors concerned: increased physical workload means higher brain concentrations and reactive metabolite formations, thus increased risk; changes in renal function have no toxicological importance in this case and therefore increase the random variability.

\section{Comments}

A population physiological model was used to describe, compare, and analyse variability in biological and air monitoring of solvent exposure. Several general conclusions may be drawn from the results obtained.

(1) When choosing between air and biological monitoring, or between several different biological indicators, exposure has to be carefully defined. It has been shown, for example, that indicators showing high residual variability when correlated with external exposure may nevertheless be excellent predictors of certain aspects of the internal exposure. The reverse is also true.

(2) The variability in the results of biological 
monitoring is not only due to individual physiological or metabolic factors. Fluctuations in exposure, both between and within days, play a large part in determining the variability in biological indicators. In certain cases such as tetrachloroethylene, this is the main factor responsible for variability.

(3) The influence of individual factors on the results of monitoring depends both on the solvent and the metabolites considered. Those influences also change according to whether the external or the internal exposure is considered.

The results obtained in the present study should be considered as predictive and not conclusive. They are based on a partially validated model ${ }^{4}$ that simulates a much simplified version of reality. Many parameters were not taken into account including:

The presence of trends or non-random fluctuations in exposure which might change considerably the conclusions concerning variability of certain end of shift indicators-notably when the end of shift and the end of exposure do not correspond.

The physical exercise done after work which may increase pulmonary excretion and therefore enhance variability in the corresponding biological indicators before shift.

The within individuals variability from day to day, which might change the results obtained, especially where one worker is repeatedly monitored.

The existence of other individual factors that could be important in certain cases, such as protein binding, dependance of renal function on urine flow rate and variability in the solubilities in tissues.

The presence under field conditions of skin resorption that will favour the use of biological indicators rather than air monitoring.

The simultaneous absorption of other chemicals, either of occupational (mixed exposure) or social origin (alcohol).
The results obtained with the present model shoutd therefore be considered as a first step towards under standing variability. They should be challenged planning further research, notably epidemiological studies for a tentative validation of the relation with the internal exposure concepts.

\section{References}

1 Gompertz D. The relationship between biological monitoriag strategies and metabolic handling. A review. Ann Occup Hyg 1981;23:405-10.

2 Hemmen JJ, de Mik G. Biological monitoring of solvents, panacea. In: Ho MH, Dillon HK, eds. Biological monitoringof exposure to chemicals. Vol I. Organic Compounds. New Yo\& John Wiley, 1987:73-84.

3 Droz PO, Berode M, Biollat MA, Lob M. Biological monitorif and health surveillance of rotogravure printing worke exposed to toluene. In: Ho MH, Dillon HK, eds. Biological monitoring of exposure to chemicals. Vol I. Organic Compound New York: John Wiley, 1987:111-31.

4 Droz PO, Wu MM, Cumberland WG, Berode M. Variability 은 biological monitoring of solvent exposure. I Development and validation of a population model. Br J Ind Med 1989;46:447-6

5 American Conference of Government Industrial Hygienis Threshold limit values and biological exposure indices chemical substances and physical agents in the work environment for 1987-88. Cincinnati: ACGIH, 1987.

6 Deutsche Forschungsgemeinschaft. Maximale Arbeitsplatzk 0 zentrationen und Biologische Arbeitsstofftoleranzwerte. Befn heim: Verlag Chemie, 1986.

7 Rappaport SM. Smoothing of exposure variability at the receptof. implication for health standards. Ann Occup Hyg 19\%; 201-14.

8 Astrand I. Uptake of solvents in the blood and tissues of mat Scand J Work Environ Health 1975;1:199-218.

9 Komiya I. Urine flow dependence of renal clearance and interre? tion of renal reabsorption and physiochemical properties $\vec{g}$ drugs. Drug Metab Dispos 1986;14:239-45.

10 Imbriani M, Ghittori S, Pezzagno G, Capodaglio E. Toluene ang styrene in urine as biological exposure indices. Appl Ind $H$ 1986;1:172-6. 